\title{
Pimavanserin
}

\section{A novel therapeutic option for Parkinson disease psychosis}

\author{
Trevor Hawkins, MD; Brian D. Berman, MD, MS
}

\begin{abstract}
Purpose of review: In April 2016, the Food and Drug Administration (FDA) approved a first-in-class atypical antipsychotic medication called pimavanserin for the treatment of Parkinson disease psychosis (PDP). We aim to inform readers about its indications, effectiveness, and safety profile. Recent findings: Pimavanserin acts as an inverse agonist at serotonin 5-HT2A receptors and has negligible effects on other receptors, thereby avoiding the D2 receptor antagonism that can potentially worsen motor symptoms. Its FDA approval was based primarily on the results of a single randomized, placebo-controlled phase 3 trial. Summary: While pimavanserin appears to be a safe, effective, and well-tolerated therapeutic option for PDP, additional clinical trials and open-label extension studies are needed to determine the long-term safety and efficacy of this promising therapy. In the meantime, prescribers need to be aware of the possible adverse effects of pimavanserin including QT interval prolongation and a potential to cause a paradoxical worsening of symptoms. Neurol Clin Pract 2017;7:157-162
\end{abstract}

$\mathrm{P}$ arkinson disease (PD) is a complex neurobehavioral disorder that makes up a substantial portion of patients seen in neurology clinics. While most are aware of its characteristic motor symptoms, the nonmotor symptoms of PD can often have more effect on quality of life and prove more difficult to treat. In particular, many patients develop hallucinations and delusions, which can occur either independently or secondary to dopaminergic medications and are frequently persistent and progressive. The PD psychosis (PDP) diagnostic criteria, as recently outlined by the National Institute of Neurological Disorders and the National Institute of Mental Health Work Group, are shown in table $1 .{ }^{1}$ Prevalence estimates suggest that up to $50 \%$ of patients with PD may be affected by psychosis, and several studies have shown that its presence is associated with functional decline, greater caregiver burden, and risk of nursing home placement, as well as increased morbidity and mortality. ${ }^{1,2}$

\section{Available treatment options}

After ruling out secondary causes of psychosis, one of the first steps to treat PDP is often an attempt to lower or change dopaminergic medications or other possible exacerbating medications (e.g., anticholinergic medications). Adjusting medication regimens in many cases, however, can lead to decreased control of motor and nonmotor symptoms, worsened quality of life,

Department of Neurology (TH, BDB), University of Colorado Anschutz Medical Campus, Aurora; and Neurology 
greater caregiver burden, and increased risk of falls. Using one of the many available typical and atypical antipsychotic treatments to treat patients with $\mathrm{PD}$ with hallucinations and psychosis may be effective, but excessive D2 dopamine receptor blockade could also lead to worsening parkinsonism. To limit potential worsening of motor symptoms, 2 of the most often frequently prescribed antipsychotic medications for PDP have been quetiapine and clozapine, which have minimal D2 receptor activity.

Clozapine, in placebo-controlled studies, has demonstrated some effectiveness in the treatment of PDP, ${ }^{3,4}$ though these studies frequently included patients with more advanced PD and dementia. The mechanism of action of clozapine for treating psychosis is not definitively known, but is thought to be a combination of D2 receptor antagonism combined with 5-HT2A receptor inverse agonism..$^{5}$ At the doses used to treat PDP, which are typically lower than those used to treat schizophrenia, clozapine has been shown to have minimal effects on D2 receptors, minimizing its risk of extrapyramidal side effects while still maintaining strong 5-HT2A inverse agonism. ${ }^{6}$ Thus, the effect of clozapine at the doses used to treat psychosis in PD could stem more from its effects on 5-HT2A activity than its antidopaminergic effects.

The use of clozapine to treat PDP is limited due to its associated potentially fatal complication of agranulocytosis and other blood cell production effects that necessitate mandatory intensive blood monitoring, ${ }^{7}$ which can place a significant burden on elderly patients and those with dementia as well as their caregivers. Other common side effects (reported in $\geq 5 \%$ ) that may limit its use in the PDP population include sedation, cardiovascular effects including orthostatic hypotension, and anticholinergic effects including constipation. ${ }^{7}$

Although quetiapine is one of the most commonly prescribed medications to treat $\mathrm{PDP},{ }^{8}$ and is generally well-tolerated at low doses, there overall is limited evidence to support its effectiveness in treating PDP. ${ }^{9}$ Quetiapine is a dibenzothiazepine and structurally similar to clozapine, but it has less inverse agonist activity at 5-HT2A, possibly explaining its less robust clinical benefit than clozapine. Findings from 2 clinical trials comparing quetiapine and clozapine head-to-head as treatments for PDP, however, suggest there may be minimal difference between these 2 treatment options. ${ }^{10,11}$ While several double-blind placebo-controlled trials have shown no significant worsening of motor scores in patients with PD, 9 quetiapine is associated with a number of potentially limiting side effects (reported in $\geq 5 \%$ ) in this patient population, including somnolence, constipation, orthostatic hypotension, and fatigue. ${ }^{12}$ These adverse effects are believed to be at least in part caused by its antagonism of H1 (somnolence) and $\alpha$-adrenergic (orthostatic hypotension) receptor affinity.

Another treatment strategy that is sometimes considered in PDP is the use of acetylcholinesterase inhibitors. In a small, open-label study of patients with PDP without dementia, donepezil use resulted in a significant decrease in psychosis. ${ }^{13}$ In a larger double-blind placebo-controlled study of 188 patients with PD with dementia and visual hallucinations, rivastigmine showed greater improvement in hallucinations compared to placebo. ${ }^{14}$ The interpretation and

Table 1 The National Institute of Neurological Disorders and Stroke and National Institute of Mental Health Working Group's diagnostic criteria for Parkinson disease psychosis $^{1}$

1. Primary diagnosis of Parkinson disease

2. Presence of at least one of the following symptoms: illusions, false sense of presence hallucinations, or delusions

3. Symptoms of psychosis occur after onset of Parkinson disease

4. Duration of symptoms of psychosis are recurrent or continuous for at least 1 month

5. Symptoms of psychosis are not accounted for by another cause such as dementia with Lewy bodies, a psychiatric disorder such as schizophrenia or mood disorder with psychotic features, or a medical condition such as delirium 


\section{Antagonism of 5-HT2 receptor activity may have particular relevance for the treatment of psychosis in PD.}

generalizability of this finding, however, is limited as the study included only patients with dementia and also permitted the concomitant use of antipsychotic medications in addition to rivastigmine. Overall, sufficient relevant data are lacking to recommend acetylcholinesterase inhibitors for the treatment of PDP.

\section{Development of pimavanserin}

Based on a long history of studies in schizophrenia and other psychotic disorders, D2 receptor antagonism has been proven to be an effective mechanism to treat psychosis. ${ }^{5}$ It has been recognized, however, that many atypical antipsychotics also share a common molecular property of serotonin 5-HT2 receptor antagonism and that this antagonism may help explain some of the antipsychotic effects and provide a novel treatment target. ${ }^{15}$ Consequently, medical researchers began searching for molecules with isolated 5-HT2A inverse agonist properties that could be used to treat psychosis and avoid the undesirable adverse effects caused by the antagonism at other receptor sites. ${ }^{16}$ The initial screening led to discovery of AC-90179, which showed isolated activity at the 5-HT2A receptors in animal studies, but ultimately development was limited by poor bioavailability. This compound subsequently led to the development of pimavanserin (ACP-103), which had similar receptor properties and much greater bioavailability. ${ }^{16}$ Pimavanserin acts as a highly selective serotonin 5-HT2A receptor inverse agonist, which works by effectively inhibiting the constitutive basal activity of the 5-HT2A receptors in addition to acting as a receptor antagonist, and negligibly binds dopaminergic, adrenergic, histaminergic, and muscarinic receptors. ${ }^{17}$

Antagonism of 5-HT2 receptor activity may have particular relevance for the treatment of psychosis in PD. Animal studies have demonstrated that there are reactive adaptations in serotonergic signaling including upregulation of 5-HT2A mRNA in the striatum in response to destruction of dopaminergic neurons. ${ }^{18}$ In humans, there is also increasing evidence that dysfunctional 5-HT2 signaling contributes to the development of psychosis in patients with PD. ${ }^{19,20}$ The fact that pimavanserin specifically targets 5 HT2A receptor activity while avoiding blockade at dopaminergic and other nonserotonergic receptors made it an ideal candidate to be tested for effectiveness in the treatment of PDP.

Indeed, in a recent multicenter, randomized, placebo-controlled phase 3 trial, pimavanserin was tested as a treatment in PDP and found to result in a 37\% improvement in psychosis as rated by the PD-adapted Scale for Assessment of Positive Symptoms (SAPS-PD), compared to $14 \%$ improvement for placebo. ${ }^{21}$ The 6-week study included 199 patients with PDP (mean age 72.4 years) who were not taking any antipsychotic medications, but were taking their typical antiparkinsonian medications as long as their regimen had been stable for 1 month. Patients in the study had to have a Mini-Mental State Examination (MMSE) score $>21 / 30$, and were excluded if taking anticholinergic medications or drugs known to prolong QT intervals, or if they had preexisting prolonged QT (see below). Secondary analyses revealed a similar level of improvement in hallucinations and delusions across all age groups, between sexes, and low and high levels of cognitive impairment. In addition, pimavanserin did not affect motor symptom severity and led to significant decreases in caregiver burden, nighttime sleep, and daytime wakefulness.

Based on the positive results of this large phase 3 trial of pimavanserin, ${ }^{21}$ and the limited evidence supporting the use of other available treatments for PDP, the Food and Drug Administration (FDA) in April 2016 approved pimavanserin (Nuplazid; Acadia Pharmaceuticals, San Diego, CA), taken as 2 tablets of $17 \mathrm{mg}$ once daily, for the treatment of hallucinations 


\section{Pimavanserin is covered on all Medicare Part D plans and is available for use at Veterans Affairs Medical Centers, and most commercial payers are now covering it.}

and delusions associated with PDP. ${ }^{17}$ Because the positive randomized, placebo-controlled trial was well-designed with clearly defined primary outcome and exclusion/inclusion criteria, and since more than $80 \%$ of enrolled participants completed the trial, it reaches Class I level of evidence. As such, it can be classified as a level B recommendation in the American Academy of Neurology classification of recommendation scheme. This is currently the same level of recommendation for clozapine, but higher than that for quetiapine (table 2). ${ }^{21,22}$

The FDA approval of a new, first-in-class medication specifically for the treatment of PDP has been greeted with much enthusiasm. There have also been some valid concerns raised. One such concern is that the approval of this medication was predominantly based on the results of a single randomized, placebo-controlled trial that was short ( 6 weeks) and had small effect sizes for all its outcome measures. In addition, there has been some concern raised that this study incorporated a psychosis scale that while it was designed to be more specific for symptoms in PDP, it has not been validated. Additional and longer duration studies are needed to validate the SAPS-PD and further support the effectiveness of pimavanserin in the treatment of PDP. Results from 2 open-label extension trials, including over 150 patients exposed to pimavanserin for over 24 months, ${ }^{23}$ should help provide key information on the long-duration response to pimavanserin.

\section{Prescribing information}

The wholesale cost of pimavanserin for a 30 -day supply is $\$ 1,950$, but the actual cost for the patient may vary considerably. Pimavanserin is covered on all Medicare Part D plans and is available for use at Veterans Affairs Medical Centers, and most commercial payers are now covering it. When prescribing pimavanserin for PDP, it is important to keep in mind its safety profile. Like other classes of antipsychotic medications, pimavanserin can prolong QT intervals (mean increase in QTc 5-8 ms with a $34 \mathrm{mg} / \mathrm{d}$ dose). Thus, its use should be avoided in patients taking other medications that prolong the QT interval, such as other antipsychotic medications, ${ }^{24}$ and in those patients with known QT prolongation or history of cardiac arrhythmias. Pimavanserin also carries a similar black box warning to other classes of antipsychotic medications of an increased risk of death in elderly patients with dementia. In the positive phase 3 trial of pimavanserin detailed above, there were 3 deaths in the treatment group and 1 in the placebo group. ${ }^{21}$ Importantly, however, all the deaths in the treated group were considered unlikely or not related to treatment.

Another potential issue to be aware of when prescribing pimavanserin is that it may induce a paradoxical worsening of psychosis in some patients. Of the 104 patients who received

Table 2 AAN classification of recommendations for treatment of Parkinson disease psychosis $^{21,22}$

Medication

Pimavanserin

Clozapine

Quetiapine

Abbreviation: AAN = American Academy of Neurology.
Current AAN classification

Level B (should be considered)

Level B (should be considered)

Level C (may be considered) 
pimavanserin in the recent phase 3 trial, 6 discontinued it due to worsening hallucinations shortly after starting the drug. Prescribers should be aware of this important potential reaction that may occur early after initiation of treatment. In addition, peripheral edema and nausea were reported in $7 \%$ of patients, a greater frequency than seen in the placebo group. Given the time course of improvement seen in the positive 6 -week phase 3 trial, ${ }^{21}$ prescribers should also be aware that the antipsychotic effects of pimavanserin may not appear until 14 days or later after initiation.

Importantly, psychosis is a common symptom seen in PD dementia and dementia with Lewy bodies. In the recent phase 3 tri$\mathrm{al}^{21}$ however, patients with PD were excluded if they had a diagnosis of dementia at baseline, limiting the ability to comment on the safety and efficacy of pimavanserin in these clinically overlapping disorders. Although a subgroup analysis dividing patients into cognitively impaired (MMSE between 21 and 24) and cognitively normal (MMSE $\geq 25$ ) showed no clinically meaningful differences in safety or effectiveness between them, ${ }^{23}$ additional studies that include patients with PD dementia and dementia with Lewy bodies are needed to determine whether pimavanserin is safe and effective in these patient populations. Furthermore, while it is currently being studied in other conditions associated with psychosis such as schizophrenia and Alzheimer dementia, pimavanserin has not been FDA-approved for any conditions other than PDP. Nevertheless, it has shown some benefit for psychosis as an adjunct to antidopaminergic therapy in schizophrenia, ${ }^{25}$ and positive results from a phase 2 double-

blind, placebo-controlled trial investigating pimavanserin to treat psychosis in Alzheimer disease have just been released (acadia-pharm.com). In this study with 181 enrolled patients, $34 \mathrm{mg}$ of pimavanserin daily led to a significant reduction in psychosis as measured by the Neuropsychiatric Inventory-Nursing Home Psychosis score at 6 weeks when compared to placebo, warranting further study in a phase 3 clinical trial.

\section{REFERENCES}

1. Ravina B, Marder K, Fernandez HH, et al. Diagnostic criteria for psychosis in Parkinson's disease: report of an NINDS, NIMH work group. Mov Disord 2007;22:1061-1068.

2. Macleod AD, Taylor KS, Counsell CE. Mortality in Parkinson's disease: a systematic review and meta-analysis. Mov Disord 2014;29:1615-1622.

3. The Parkinson Study Group. Low-dose clozapine for the treatment of drug-induced psychosis in Parkinson's disease.N Engl J Med 1999;340:757-763.

4. The French Clozapine Parkinson Study Group. Clozapine in drug-induced psychosis in Parkinson's disease.Lancet 1999;353:2041-2042.

5. Fakra E, Azorin JM. Clozapine for the treatment of schizophrenia. Expert Opin Pharmacother 2012; 13:1923-1935.

6. Meltzer HY, Kennedy J, Dai J, Parsa M, Riley D. Plasma clozapine levels and the treatment of L-DOPA-induced psychosis in Parkinson's disease: a high potency effect of clozapine. Neuropsychopharmacology 1995;12:39-45.

7. Clozaril [package insert]. Rosemont, PA: HLS Therapeutics, Inc.; 2016.

8. Weintraub D, Chen P, Ignacio RV, Mamikonyan E, Kales HC. Patterns and trends in antipsychotic prescribing for Parkinson disease psychosis. Arch Neurol 2011;68:899-904.

9. Desmarais P, Massoud F, Filion J, Nguyen QD, Bajsarowicz P. Quetiapine for psychosis in Parkinson disease and neurodegenerative parkinsonian disorders: a systematic review. J Geriatr Psychiatry Neurol 2016;29:227-236.

10. Morgante L, Epifanio A, Spina E, et al. Quetiapine and clozapine in parkinsonian patients with dopaminergic psychosis. Clin Neuropharmacol 2004;27:153-156. 
11. Merims D, Balas M, Peretz C, Shabtai H, Giladi N. Rater-blinded, prospective comparison: quetiapine versus clozapine for Parkinson's disease psychosis. Clin Neuropharmacol 2006;29:331-337.

12. Seroquel [package insert]. Wilmington, DE: AstraZeneca Pharmaceuticals, LP.; 2009.

13. Fabbrini G, Barbanti P, Aurilia C, Pauletti C, Lenzi GL, Meco G. Donepezil in the treatment of hallucinations and delusions in Parkinson's disease. Neurol Sci 2002;23:41-43.

14. Burn D, Emre M, McKeith I, et al. Effects of rivastigmine in patients with and without visual hallucinations in dementia associated with Parkinson's disease. Mov Disord 2006;21:1899-1907.

15. Sullivan LC, Clarke WP, Berg KA. Atypical antipsychotics and inverse agonism at 5-HT2 receptors. Curr Pharm Des 2015;21:3732-3738.

16. Hacksell U, Burstein ES, McFarland K, Mills RG, Williams H. On the discovery and development of pimavanserin: a novel drug candidate for Parkinson's psychosis. Neurochem Res 2014;39:2008-2017.

17. Vanover KE, Weiner DM, Makhay M, et al. Pharmacological and behavioral profile of N-(4fluorophenylmethyl)-N-(1-methylpiperidin-4-yl)-N'-(4-(2-methylpropyloxy)phen ylmethyl) carbamide (2R,3R)-dihydroxybutanedioate (2:1) (ACP-103), a novel 5-hydroxytryptamine(2A) receptor inverse agonist. J Pharmacol Exp Ther 2006;317:910-918.

18. Zhang X, Andren PE, Svenningsson P. Changes on 5-HT2 receptor mRNAs in striatum and subthalamic nucleus in Parkinson's disease model. Physiol Behav 2007;92:29-33.

19. Ballanger B, Strafella AP, van Eimeren T, et al. Serotonin 2A receptors and visual hallucinations in Parkinson disease. Arch Neurol 2010;67:416-421.

20. Huot P, Johnston TH, Darr T, et al. Increased 5-HT2A receptors in the temporal cortex of parkinsonian patients with visual hallucinations. Mov Disord 2010;25:1399-1408.

21. Cummings J, Isaacson S, Mills R, et al. Pimavanserin for patients with Parkinson's disease psychosis: a randomised, placebo-controlled phase 3 trial. Lancet 2014;383:533-540.

22. Seppi K, Weintraub D, Coelho M, et al. The movement disorder society evidence-based medicine review update: treatments for the non-motor symptoms of Parkinson's disease. Mov Disord 2011;26 (suppl 3):S42-S80.

23. NUPLAZID [package insert]. San Diego, CA: ACADIA Pharmaceuticals, Inc.; 2016.

24. Ballard C, Isaacson S, Mills R, et al. Impact of current antipsychotic medications on comparative mortality and adverse events in people with Parkinson disease psychosis. J Am Med Dir Assoc 2015; 16:898.e1-898.e7.

25. Meltzer HY, Elkis H, Vanover K, et al. Pimavanserin, a selective serotonin (5-HT)2A-inverse agonist, enhances the efficacy and safety of risperidone, $2 \mathrm{mg} /$ day, but does not enhance efficacy of haloperidol, 2mg/day: comparison with reference dose risperidone, 6mg/day. Schizophr Res 2012;141:144-152.

Received December 8, 2016. Accepted in final form February 2, 2017.

\section{AUTHOR CONTRIBUTIONS}

T. Hawkins: drafting/revising the manuscript. B.D. Berman: drafting/revising the manuscript, study concept or design.

\section{STUDY FUNDING}

No targeted funding reported.

\section{DISCLOSURES}

T. Hawkins reports no disclosures. B.D. Berman serves on scientific advisory boards for Benign Essential Blepharospasm Research Foundation and National Spasmodic Torticollis Association; has received funding for travel from Movement Disorder Society and Benign Essential Blepharospasm Research Foundation; and receives research support from NIH, Colorado Translational Research Imaging Center, University of Colorado Center for Neuroscience, The Dana Foundation (The David Mahoney Neuroimaging Program), and Benign Essential Blepharospasm Research Foundation, Inc. Full disclosure form information provided by the authors is available with the full text of this article at Neurology.org/cp. 